\title{
Seroprevalence of Toxoplasma gondii in Chickens (Gallus domesticus) in Sudan
}

\author{
Mohammed Osman Hussien,, ${ }^{1,}$ Shima Hassan Alfaki, ${ }^{2}$ and Abdel Rahim Mohamed El Hussein ${ }^{2}$ \\ ${ }^{1}$ Department of Microbiology, Central Laboratory, Ministry of Higher Education and Scientific Research, Khartoum, Sudan \\ ${ }^{2}$ Central Veterinary Research Laboratory (CVRL), Animal Resources Research Corporation (ARRC), El Amarat, Khartoum, Sudan \\ "Corresponding author: Mohammed Osman Hussien, Department of Microbiology, Central Laboratory, Ministry of Higher Education and Scientific Research, Khartoum, \\ Sudan. Tel: +249-122099533, E-mail: moh200432@hotmail.com
}

Received 2016 June 23; Accepted 2016 July 03.

\begin{abstract}
Background: Toxoplasma gondii (T. gondii) infections are widely prevalent in human beings and animals worldwide. Humans become infected post-natally by ingesting tissue cysts from undercooked meat, consuming food or drink contaminated with oocysts or by accidentally ingesting oocysts from the environment.

objectives: The present study was conducted to determine the seroprevalence of T. gondii infections in chickens in Sudan, during the period between December 2015 and January 2016.

Methods: Sera were separated from blood samples, which were collected from the wing vein of chickens $(\mathrm{n}=58)$. Commercial serum agglutination kits (toxoplasmosis latex test kit) for Toxoplasma antibodies detection, using diluted blood serum samples $(1 / 2,1 / 4,1 / 8,1 / 16,1 / 32,1 / 64)$, were used.

Results: Twenty-five, seventeen and sixteen chicken sera from river Nile, Khartoum and Sennar states, were positive, respectively. These represented a seroprevalence of $100 \%$ in all states with different titers.

Conclusions: Toxoplasma gondii infection is prevalent in chickens of Sudan. Therefore, further investigations on the parasite in other States of Sudan is important.
\end{abstract}

Keywords: T. gondii, Chickens, LAT, Sudan

\section{Background}

Toxoplasma gondii (T. gondii) infections are widely prevalent in human beings and animals worldwide $(1,2)$. Humans become infected post-natally by ingesting tissue cysts from undercooked meat, consuming food or drink contaminated with oocysts or by accidentally ingesting oocysts from the environment. Felids are the most important host in the life cycle of T. gondii because they excrete environmentally resistant oocysts. Toxoplasmosis can rarely cause clinical disease in chickens (3). Historically, toxoplasmosis was first described by Hepding (4) in a hen from Germany. Microscopically, sciatic nerve neuritis, chorioretinitis and encephalitis were the main lesions. Hepding (4) proposed a new name, Toxoplasma gallinarum, for the organism in this hen. This case was considered to be a primary infection with Marek's disease virus with secondary involvement of toxoplasmosis

\section{Objectives}

Owing to the fact that chickens are considered one of the important hosts in the epidemiology of T. gondii infection (3). The present study was conducted to determine the seroprevalence of T. gondii infections in chickens of Sudan, during the period between December 2015 and January 2016.

\section{Methods}

Sera were separated from blood samples, which were collected from the wing vein of chickens $(\mathrm{n}=58)$. Commercial serum agglutination kits (toxoplasmosis latex test kit) for toxoplasma antibodies detection using diluted blood serum samples $(1 / 2,1 / 4,1 / 8,1 / 16,1 / 32,1 / 64)$ were purchased from Plasmatec, (UK). The test was performed according to the manufacturer's instructions. Any clear agglutination indicates the presence of Toxoplasma antibodies equal or greater than $4 \mathrm{IU} / \mathrm{mL}$, which reflects either a past infection or an evolving infection. Sensitivity of the test is $3-7 \mathrm{IU} / \mathrm{mL}$.

\section{Results}

Twenty-five, seventeen and sixteen chicken sera from river Nile, Khartoum and Sennar States, were positive, respectively. These represented a seroprevalence of $100 \%$ in all states with different titers (Table 1).

\section{Discussion}

To the best of our knowledge, this is the first report on the presence of T. gondii antibodies in chickens of Sudan. In the present study, T. gondii antibodies were detected in free-range (FR) chickens in river Nile and Sennar States. Toxoplasma gondii infection in free-range chickens is considered important, as free-range chickens are one of the best indicators for soil contamination with T. gondii oocysts, because they feed from the ground, and tissues of infected 
Table 1. Seroprevalence of Toxoplasma gondii Infection in Chickens From Three States in Sudan Using LAT

\begin{tabular}{|c|c|c|c|c|c|c|c|c|c|}
\hline \multirow[t]{2}{*}{ State } & \multirow[t]{2}{*}{ Source of Chicken } & \multirow[t]{2}{*}{ No. Chickens Tested } & \multirow[t]{2}{*}{ No. Positive (\%) } & \multicolumn{6}{|c|}{ No. Chicken With LAT Titers } \\
\hline & & & & 2 & 4 & 8 & 16 & 32 & 64 \\
\hline River Nile & FR & 25 & $25(100)$ & - & 1 & 1 & 5 & 5 & 13 \\
\hline Khartoum & c & 17 & $17(100)$ & - & 2 & 1 & 4 & 5 & 5 \\
\hline Sennar & FR & 16 & $16(100)$ & . & . & . & 2 & 7 & 7 \\
\hline
\end{tabular}

Abbreviations: C, commercial farm; $\mathrm{FR}$, free-range.

chickens are considered a good source of infection for cats (3). Moreover, ingestion of infected chicken's meat can be a source of infection for T. gondii for humans and other animals. Therefore, FR chickens play an important role in the epidemiology of $T$. gondii in the rural environment, perhaps more than rodents, because they are clinically resistant to T. gondii and live longer than rodents (3). The $T$. gondii prevalence of $20 \%, 32 \%$ and $57.5 \%$ was reported in Sudanese camels, cattle and sheep, respectively (5). Elfahal et al. (6) reported that the infection rate of $T$. gondii in cattle at the herd level in Khartoum State was 50\% whereas this was 33.3\% in Gezira state. The prevalence rate of T. gondii infection in the Sudanese subjects ranges from 20.2 to $70 \%$. Most of the surveys were conducted on pregnant women from Khartoum and Gezira States (7). Our finding may pave the way towards exploring the risk of human infection with $T$. gondii through the consumption of undercooked infected chicken meat. It could be concluded that T. gondii infection is prevalent in chickens of Sudan. Therefore, further investigation on the parasite in other States of Sudan is important. Moreover, confirmation of the current results requires the isolation of viable $T$. gondii from chickens using bioassay in mice.

\section{Acknowledgments}

The authors wish to thank chicken farm owners for permission to collect the samples.

\section{Footnotes}

Authors' Contribution: Mohammed Osman Hussien and Shima Hassan Alfaki conducted the sample collection and Latex agglutination test; Abdel Rahim Mohamed El Hussein contributed to the concept and design of the study as well as revision of the manuscript.

Funding/Support: This study was supported by the central laboratory, ministry of higher education and scientific research, Khartoum, Sudan

\section{References}

1. Dubey JP, Beattie CP. Toxoplasmosis of animals and man. CRC Press. 1988:1-220.

2. Dubey JP. Toxoplasmosis of animals and humans: CRC. 2009

3. Dubey JP. Toxoplasma gondii infections in chickens (Gallus domesticus): prevalence, clinical disease, diagnosis and public health significance. Zoonoses Public Health. 2010;57(1):60-73. doi: 10.1111/j.18632378.2009.01274.x. [PubMed: 19744305].

4. Hepding L. Ueber Toxoplasmen (Toxoplasma gallinarum n. sp.).Z.f. Infektionskrankheiten der Haustiere. 1939;55:109-16.

5. Khalil MK, Intisar E. Seroprevalence of Toxoplasma gondii antibodies in farm animals (camels, cattle, and sheep) in Sudan. J Vet Med Anim Health. 2011;3:36-9.

6. Elfahal AM, Elhassan AM, Hussien MO, Enan KA, Musa AB, El Hussein AM. Seroprevalence of Toxoplasma gondii in Dairy Cattle with Reproductive Problems in Sudan. ISRN Vet Sci. 2013;2013:895165. doi: 10.1155/2013/895165. [PubMed: 24171116].

7. Ibrahim AM, Abakar AD. Human Toxoplasmosis in the Sudan: A review. Eur Acad Res. 2015;3(6):6890-904. 